\title{
Co-Occurrence of Chronic Spontaneous Urticaria with Immunoglobulin A Deficiency and Autoimmune Diseases
}

\author{
Barbara Frossi ${ }^{a, b}$ Stefano De Carlic Fleur Bossi ${ }^{e}$ Carlo Pucillo $^{a, b}$ \\ Marco De Carlic, d \\ ${ }^{a}$ Dipartimento di Scienze Mediche e Biologiche and ${ }^{\mathrm{b}} \mathrm{MATI}$ Center of Excellence, Università degli Studi di Udine, and \\ cAmbulatorio Malattie Rare and d Allergologia e Immunologia Clinica, SOC Medicina 2, Dipartimento di Medicina

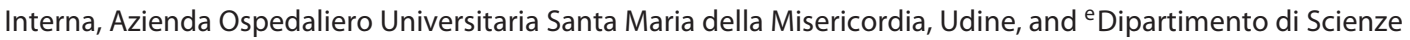 \\ Chirurgiche, Mediche e della Salute, Università di Trieste, Trieste, Italy
}

\section{Key Words}

Chronic spontaneous urticaria $\cdot$ Immunoglobulin A

deficiency · Autoimmunity · Mast cells $\cdot$ Thyroiditis · Vitiligo

\begin{abstract}
Background: Immunoglobulin (lg) A deficiency is a primary immunodeficiency in which autoimmunity is frequently observed. Thirty to fifty percent of patients with spontaneous chronic urticaria have autoantibodies that are able to crosslink FceRI on mast cells and basophils. Methods: We investigated whether spontaneous chronic urticaria in patients with IgA deficiency meets the criteria for autoimmunity. Four patients were screened for positivity to a skin prick test and an autologous serum skin test and for the presence of other autoimmune diseases. Patient sera were tested for the ability to activate basophils and mast cells in vitro by measuring surface CD63 expression and $\beta$-hexosaminidase release, respectively. Results: The autologous serum test was positive in all patients, and patient sera were found to induce CD63 upregulation on basophils and degranulation of an LAD2 mast cell line. Moreover, all patients were affected
\end{abstract}

\section{KARGER}

E-Mail karger@karger.com

www.karger.com/iaa by other autoimmune disorders. Conclusion: For the first time, these data point out chronic autoimmune urticaria in subjects with an IgA deficiency and confirm that different autoimmune disorders are common among patients with an IgA deficiency. Patients with chronic autoimmune spontaneous urticaria should be screened for IgA deficiency, especially if they are affected by other autoimmune disorders. Thus, spontaneous urticaria could mirror more complex systemic diseases, such as immune deficiency.

(c) 2016 S. Karger AG, Basel

\section{Introduction}

Chronic spontaneous urticaria (CSU) affects about $0.1 \%$ of the population, with a higher incidence in women and a peak age between 20 and 40 years [1]. One fourth of patients with CSU display autoantibodies against thyroid autoantigens [2], while $40 \%$ of patients show antibodies against immunoglobulin (Ig) E or its high-affinity receptor FceRI that induce histamine release by mast cells (MC) and basophils [3]. Current guidelines for the diag-
(C) 2016 S. Karger AG, Basel

$1018-2438 / 16 / 1692-0130 \$ 39.50 / 0$ 
Table 1. Main clinical and laboratory features of the patients

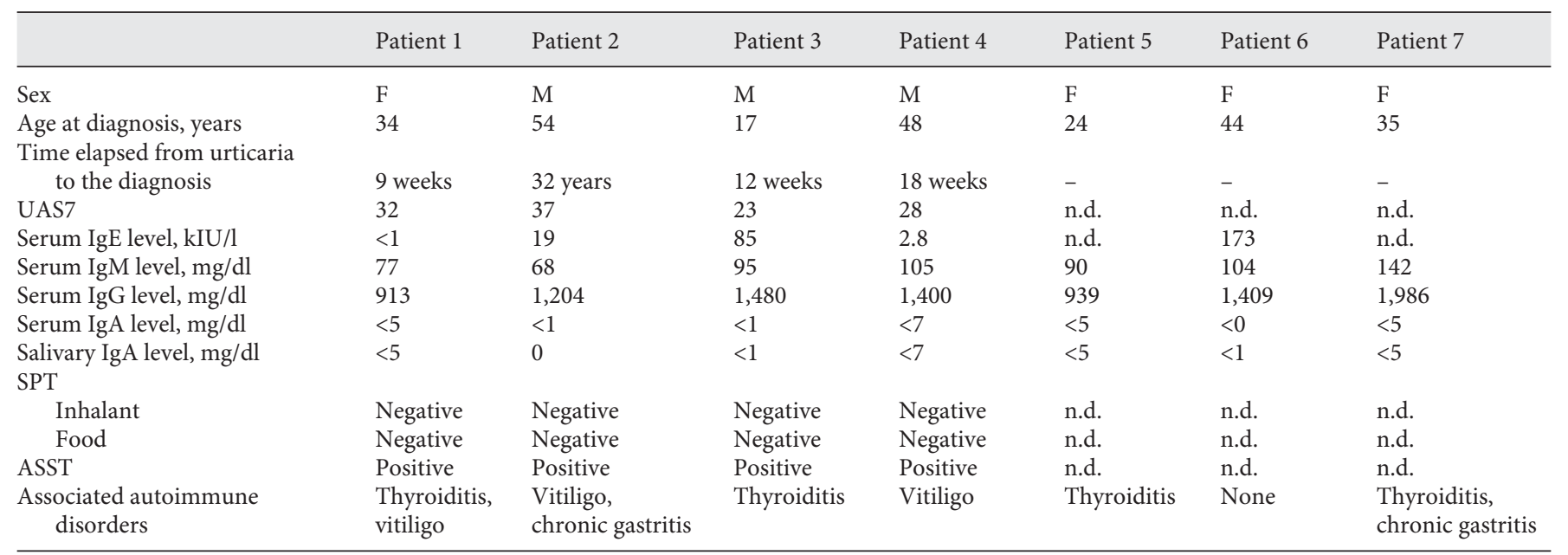

Normal values: IgM, 65-210 mg/dl; IgG, 740-1,440 mg/dl; serum IgA, 140-400 mg/dl; salivary IgA, 5-20 mg/dl; IgE, 1.5-100 kIU/l. The ASST was considered positive when the red wheal had a diameter at least $1.5 \mathrm{~mm}$ greater than with the control saline solution. $\mathrm{n} . \mathrm{d}$. $=\mathrm{Not}$ done; $\mathrm{M}=$ male; $\mathrm{F}=$ female.

nosis of CSU still recommend the autologous serum skin test (ASST) to define an autoimmune etiology [4] even if its accuracy and reliability are still debated and controversial. Evaluation of autoantibodies against IgE or FceRI, as well as the basophil activation test (BAT), can also be performed to confirm serum autoreactivity. However, no correlation between ASST results and autoantibodies has been proven, and not all antibodies have a pathogenic role, suggesting that other as yet unidentified factors are responsible for the development of wheals and angioedema [5]. Thus, CSU is not a single disease but it is rather a pattern of reactions that reflect cutaneous MC degranulation. Indeed, we have previously demonstrated that in vitro evaluation of $\mathrm{MC}$ reactivity to CSU serum could represent a tool for CSU patient screening [6].

IgA deficiency (IGAD) is the most common primary immunodeficiency (PID), defined as a serum IgA level $\leq 0.07 \mathrm{~g} / \mathrm{l}$ with or without an IgA secretory defect and with normal levels of serum IgG and IgM in patients older than 4 years in whom other causes of hypogammaglobulinemia have been excluded [7]. Compared to healthy individuals, patients with IGAD have a greater risk of concomitant autoimmune disorders [8].

The incidence of skin manifestations in IGAD has only been scarcely investigated. Recently, Gualdi et al. [9] carried out and published a longitudinal prospective study in 147 patients with IGAD on several skin manifestations ranging from atopic dermatitis, psoriasis, and vitiligo to skin infection. However, the incidence of CSU and the correlation of CSU and IGAD were not considered in that study. Indeed, no data are available to date regarding the relationship between IgA levels and CSU.

Herein, we report 4 patients with autoimmune CSU associated with IGAD.

\section{Materials and Methods}

\section{Patients}

Patients were selected among those attending the Second Unit of Internal Medicine of the Azienda Ospedaliero Universitaria of Udine. Disease activity was estimated as the urticaria activity score (UAS) according to European Academy of Allergy and Clinical Immunology (EAACI) guidelines [4]. The UAS7 (weekly UAS) was assessed on the basis of symptoms evaluated the week before performance of the ASST. Subjects gave informed consent for participation in this study. At the time of the experiments, the patients had been off medication for at least 5 days.

\section{Basophil Activation Test}

Skin prick tests (SPT) were carried out using commercial extracts by Lofarma Allergeni S.p.A. (Milan, Italy) and ALK-Abellò (Hørsholm, Denmark). The ASST was performed via intradermal injection of $0.05 \mathrm{ml}$ fresh autologous serum and reading of the wheal-and-flare reaction after $30 \mathrm{~min}$ [10]. Basophil activation was evaluated using a FastImmune ${ }^{\mathrm{TM}}$ CD63/CD123/anti-HLA-DR kit following the manufacturer's indications (Becton Dickinson, San Jose, Calif., USA).

\section{Degranulation Assay}

Evaluation of MC degranulation was performed using the human MC line LAD2 as previously published [6]. Briefly, $2 \times 10^{5}$ 


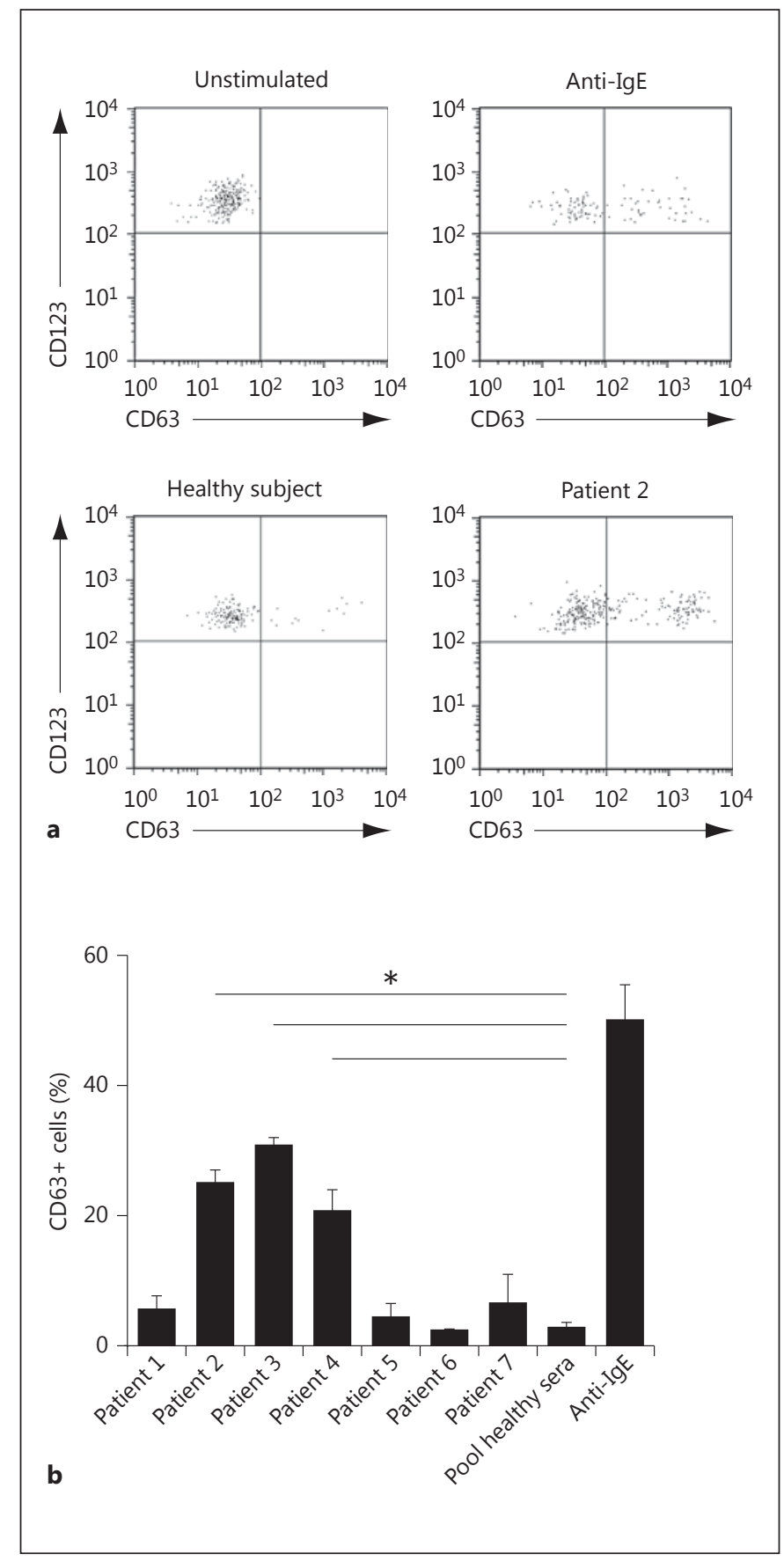

Fig. 1. BAT. Peripheral blood was incubated with an equal volume of serum from patients or healthy donors. a Degranulated basophils were identified as CD63+/CD123+ cells. Polyclonal anti-IgE was used as the positive control. Representative plots are shown. b The means \pm SD of the CD63+/CD123+ cell percentages from 3 independent experiments for each condition are shown. The mean of percentages obtained with healthy sera is shown (pool healthy sera). ${ }^{*} \mathrm{p}<0.001$.
LAD2 cells were incubated with $2 \mu \mathrm{l}$ serum in Tyrode's buffer for $30 \mathrm{~min}$ at $37^{\circ} \mathrm{C}$, and the extent of degranulation was measured as the amount of released $\beta$-hexosaminidase.

Sera from 6 sex- and age-matched healthy subjects were used in both BAT and MC degranulation assays as controls. Data were compared via an ANOVA test using a post hoc analysis for paired multiple comparisons with a protected Fisher's least significant difference test.

\section{Results}

The clinical features of the patients are listed in table 1. In all patients, serum IgG and IgM levels were normal, but serum and salivary IgA were absent. In CSU patients, SPT were negative for both inhalant and food allergens.

Patient 1 was a 34-year-old woman complaining of urticaria in the last 9 weeks. The UAS7 was 32, and symptoms were controlled only by a daily intake of desloratadine and prednisone. The patient was known to be affected by Hashimoto's thyroiditis and vitiligo. Serological screening was negative for the presence of other autoimmune diseases.

Patient 2 was a 54-year-old male with vitiligo who had suffered from CSU since the age of 22 years. The urticaria was controlled by a daily intake of cetirizine and prednisone. The UAS7 was 37. Screening for autoimmune markers showed autoantibodies against parietal cells (but not autoantibodies against intrinsic factor). Chronic gastritis was confirmed by endoscopy.

Patient 3 was a 17-year-old man affected by chronic thyroiditis with symptoms of urticaria during the last 12 weeks. The UAS7 was 23 and symptoms were controlled by a daily intake of high doses of rupatadine. Screening for autoimmune markers showed IgG autoantibodies against transglutaminase, but an endoscopic biopsy was negative for celiac disease. Moreover, the patient was negative for expression of HLA DQ2 and DQ8 alleles.

Patient 4 was a 48 -year-old man with vitiligo and symptoms of urticaria in the last 16 weeks. The UAS7 was 28 and symptoms were controlled by a daily intake of a quadruple dose of desloratadine and short courses of deflazacort. In the sera, low levels of anti-nuclear antibodies with a nucleolar pattern were detected, but there were no symptoms and/or signs of overt disease.

All patients complained of angioedema while not on therapy. Patient 3 reported only few episodes limited to the lips.

As controls, we selected 3 subjects with IGAD with no complaints of urticaria (patients 5,6, and 7). In patients 


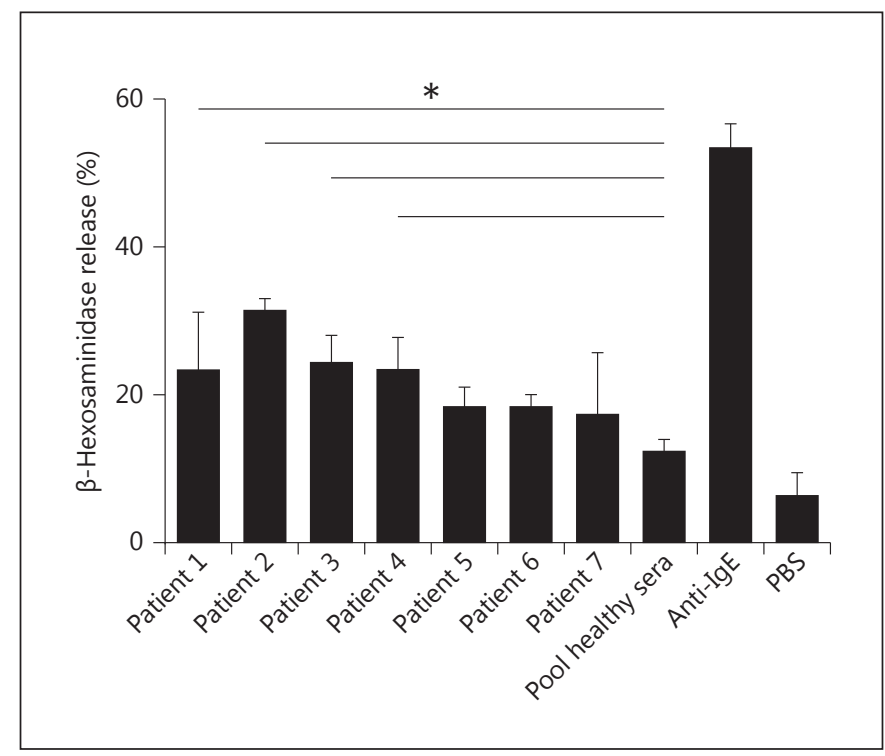

Fig. 2. MC degranulation response. LAD2 cells were incubated with sera from patients and healthy subjects. Ionomycin and PSB were used as positive and negative controls, respectively. The degranulation response was measured as the amount of released $\beta$-hexosaminidase. Data are the means of 3 independent experiments. The mean of $\beta$-hexosaminidase release obtained with healthy sera is shown (pool healthy sera). ${ }^{*} \mathrm{p}<0.001$.

5 and 7 , autoimmune disorders were diagnosed. Both had autoimmune thyroiditis and patient 7 was also affected by chronic gastritis.

To characterize the autoimmune nature of urticaria, sera from all IGAD patients were used for ASST and BAT and to measure MC reactivity. In all CIU patients, the ASST was positive. To confirm autoimmune CSU, patient sera were used to stimulate donor basophils in an in vitro heterologous assay (fig. 1). CD63, detectable on cell membranes only after activation, increased in blood basophils after incubation with all sera from the CSU patients, except for the serum of patient 1 . The sera of patients 5, 6, and 7, which were not affected by CSU, showed no significant upregulation of CD63 expression, similarly to the sera obtained from the controls. In order to further demonstrate the autoreactivity of the sera from the CSU patients, we analyzed the stimulatory effect of sera on the human MC cell line LAD2 that releases $\beta$-hexosaminidase, once activated [6]. As shown in figure 2, the sera of all 4 CSU patients (sera 1,2,3, and 4) induced significant degranulation of LAD2. The sera from patients 5,6 , and 7 did not induced a release of $\beta$-hexosaminidase as the sera from the healthy controls did.

\section{Discussion}

Diagnosis of autoimmune CSU is difficult because the clinical symptoms of CSU are the expression of a multifaceted inflammatory disease, the pathogenic mechanisms of which are still unclear [5]. ASST seems to be a useful and reliable screening test, but additional confirmatory methods are needed $[10,11]$. Basophil histamine release and BAT have been used to identify patients with CSU [12], but they yield a positive result only in a fraction of the patients [13]. We have previously demonstrated that CSU patient sera induce degranulation of human MC lines in both an FceRI-dependent and an FceRI-independent manner also in the absence of positivity to ASST [6].

In this paper, we characterized the sera of 4 IGAD patients for positivity to the ASST, the BAT, and the MC degranulation assay. Taken together, our results are consistent with the ability of sera to activate basophils and MC causing wheals and angioedema.

The association between autoreactivity in CSU and other autoimmune diseases is well established [1], and current guidelines suggest CSU patient screening for thyroid autoimmunity [4]. IGAD is a PID and its incidence varies according to geographical region. Paradoxically, a substantial proportion of PID patients develop autoimmune phenomena besides an increased susceptibility to infections because of their impaired immunity. Eightyfive to 90 percent of IGAD subjects may be asymptomatic, and autoimmunity may be the only clinical feature of this PID [7]. It has been proven that the IGAD prevalence increases in patients with autoimmune diseases, and there are high prevalence rates of autoimmune disease in IGAD patients [14]. Among autoimmune disorders, thyroiditis, vitiligo, and hemolytic anemia seem to be the most common manifestations [8].

For the first time, we described subjects with CSU due to autoimmunity and selective IGAD in the absence of evidence of allergy (i.e. normal IgE levels and negative SPT); autoimmune disorders were once again confirmed to be common in patients with IGAD.

The reasons for these associations and this predisposition are unknown, although genetically linked susceptibilities are supposed to play a role $[14,15]$. Therefore, it is known that mucosal resident bacteria shape the IgA repertoire and that the interaction between microbiota and IgA could be critical in controlling the pathogenesis of autoimmune disorders [16]. Thus, defects in IgA regulation by perturbation of the microbial composition and the gut microenvironment have the potential to increase autoimmune susceptibility.

Int Arch Allergy Immunol 2016;169:130-134 DOI: $10.1159 / 000445058$ 
Evaluation of IgA levels could be useful in terms of morbidity and follow-up of other autoimmune disorders, in addition to identification of a defect of the immune system that may predispose the individual to recurrent gastrointestinal and respiratory infections.

In this study, only few patients were described because IGAD subjects are occasionally diagnosed and IgA levels are not routinely tested in the presence of signs of CSU. However, our preliminary observations indicated that the skin manifestations can mirror more complex underlying diseases and that patients with skin autoimmune disorders should be screened for the presence of other autoimmune conditions as well as for associated immune defects.

\section{Acknowledgement}

The authors are grateful to Dr. Caterina Degano Massimo for the editing of the manuscript. This study was supported in part by the Italian Society of Mastocytosis (ASIMAS).

\section{References}

1 Maurer M, Weller K, Bindslev-Jensen C, Giménez-Arnau A, Bousquet PJ, Bousquet J, Canonica GW, Church MK, Godse KV, Grat$\tan$ CE, Greaves MW, Hide M, Kalogeromitros D, Kaplan AP, Saini SS, Zhu XJ, Zuberbier T: Unmet clinical needs in chronic spontaneous urticaria: a GA ${ }^{2} \mathrm{LEN}$ task force report. Allergy 2011;66:317-330.

2 Bagnasco M, Minciullo PL, Schiavo M, Saraceno G, Gangemi S, Benvenga S: Urticaria and thyroid autoimmunity. Thyroid 2011;21: 401-410.

3 Sabroe RA, Fiebiger E, Francis DM, Maurer D, Seed PT, Grattan CE, Black AK, Stingl G, Greaves MW, Barr RM: Classification of antiFcepsilonRI and anti-IgE autoantibodies in chronic idiopathic urticaria and correlation with disease severity. J Allergy Clin Immunol 2002;110:492-499.

4 Zuberbier T, Aberer W, Asero R, et al: The EAACI/ GA ${ }^{2} \mathrm{LEN} / \mathrm{EDF} / \mathrm{WAO}$ guideline for the definition, classification, diagnosis, and management of urticaria: the 2013 revision and update. Allergy 2014;69:868-887.

5 Fiebiger E, Maurer D, Holub H, Reininger B, Hartmann G, Woisetschlager M, et al: Serum IgG autoantibodies directed against the alpha chain of Fc epsilon RI: a selective marker and pathogenetic factor for a distinct subset of chronic urticaria patients? J Clin Invest 1995; 96:2606-2612.
6 Bossi F, Frossi B, Radillo O, Cugno M, Tedeschi A, Riboldi P, Asero R, Tedesco F, Pucillo C: Mast cells are critically involved in serummediated vascular leakage in chronic urticaria beyond high-affinity IgE receptor stimulation. Allergy 2011;66:1538-1545.

7 Yel L: Selective IgA deficiency. J Clin Immunol 2010;30:10-16.

8 Abolhassani H, Gharib B, Shahinpour S, Masoom SN, Havaei A, Mirminachi B, Arandi N, Torabi-Sagvand B, Khazaei HA, Mohammadi J, Rezaei N, Aghamohammadi A: Autoimmunity in patients with selective IgA deficiency. J Investig Allergol Clin Immunol 2015;25:112-119.

9 Gualdi G, Lougaris V, Baronio M, Vitali M, Tampella G, Moratto D, Tanghetti P, Monari P, Calzavara-Pinton P, Plebani A: Burden of skin disease in selective IgA deficiency and common variable immunodeficiency. J Investig Allergol Clin Immunol 2015;25:369-371.

10 Sabroe RA, Grattan CE, Francis DM, Barr RM, Kobza Black A, Greaves MW: The autologous serum skin test: a screening test for autoantibodies in chronic idiopathic urticaria. Br J Dermatol 1999;140:446-452.
11 Konstantinou GN, Asero R, Maurer M, Sabroe RA, Schmid-Grendelmeier P, Grattan CE: EAACI/ GA²LEN task force report: the autologous serum skin test in urticaria. Allergy 2009;64:1256-1268.

12 Szegedi A, Irinyi B, Gál M, Hunyadi J, Dankó K, Kiss E, Sipka S, Szegedi G, Gyimesi E: Significant correlation between the CD63 assay and the histamine release assay in chronic urticaria. Br J Dermatol 2006;155: 67-75.

13 Grattan CE, Francis DM, Hide M, Greaves MW: Detection of circulating histamine releasing autoantibodies with functional properties of anti-IgE in chronic urticaria. Clin Exp Allergy 1991;21:695-670.

14 Singh Karmtej, Chang Christopher, Gershwin ME: IgA deficiency and autoimmunity. Autoimmun Rev 2014;13:163-177.

15 Jorgensen GH, Thorsteinsdottir I, Gudmundsson S, Hammarstrom L, Ludviksson BR: Familial aggregation and autoimmunity. Clin Imunol 2009;131:233-239.

16 Ruff EW, Kriegel MA: Autoimmune host-microbiota interactions at barrier sites and beyond. Trends Mol Med 2015;21:233-244. 